\title{
Grains on the chessboard
}

\section{Recent progress in mapping quantitative growth traits (QTLs) in rice yields insights into mechanisms of plant growth, hints at genomic signatures of the domestication process and promotes the prospect of agricultural improvement via introgression of beneficial variants.}

$\mathrm{n}$ the legend from Ambalappuzha, Kerala, a king loses a game of chess and pays the sage who played with him

in grains of rice, doubling on each successive square of the board. This geometric process sums to $2^{64}-1$ grains, an amount equal to tens of thousands of years of today's global rice harvest (currently some 29.3 million tons according to the International Grains Council).

Plant geneticists seeking ever increasing yield one gene at a time would be delighted to find 64 gene variants that each double yield. In rice, alleles of relatively large effect (and with consistent or negligible gene-environment interaction) can indeed be used to bring advantageous combinations of quantitative traits into an elite crop variety (by a process termed QTL pyramiding: Takeda and Matsuoka, Nat. Rev. Genet.9, 444-457; 2008).

On (p 1273) Yonghong Wang and Jiayang Li review recent successes in mapping rice mutations and QTLs. Zhuhua $\mathrm{He}$ and colleagues identify a gene contributing to strain differences in grain-filling that encodes a cell wall invertase ( $p$ 1370). Chuanqing Sun and colleagues (p 1360) and Hong-Xuan Lin and colleagues ( $p$ 1365) report a gene that determines upright or prostrate growth habit.

All three genes show strain diversity consistent with selection upon these loci during agricultural domestication, confirming that one current strategy-screening for genomic signatures of selection-is likely to be generally fruitful. However, as Wang and Li point out, the process of domestication is far from a simple process of universal fixation of beneficial trait variants. For example, tiller angle and plant morphology should be under balancing selection, as cultivation imposes opposing constraints on space versus yield and disease resistance. We might therefore expect there to be economically interesting trait loci and mechanistically informative growth genes with even more complex patterns of variation.

Once QTLs have been identified, it is now common to sequence the locus across diverse isolates to identify naturally occurring alleles. An obvious application of this approach would be to extend the allelic range artificially via transgenes (if this meets with public acceptance), or to engineer allelic combinations by conventional marker-assisted breeding.

Jung et al. (Nat. Rev. Genet. 9, 91-101; 2008) emphasize that map-based cloning remains extremely labor-intensive and that genetic analysis in rice is complicated by genomic redundancy. Duplicated genomic segments cover a large proportion of the rice genome, so that high-throughput functional genomics approaches are needed in addition to genetic mapping. At $389 \mathrm{Mb}$ (Nature 436, 793-800; 2005), the rice genome is small enough to be an attractive prospect for sequencing projects to characterize whole-genome diversity. When isolates from appropriately chosen regions and cultivation types are available, the complexity of the agricultural interaction of human and crop genomes will begin to emerge and putative patterns of selection can be understood with relevant genomic controls. Combinations of alleles corresponding to a variety of economically valuable regional adaptations may then be available.

In the legend, the king is allowed to stop doubling and pay his debt of rice at a sustainable rate in a locally valuable way to all who need feeding. 